\title{
Rice Glycosyltransferase Gene UGT85E1 Is Involved in Drought Stress Tolerance Through Enhancing Abscisic Acid Response
}

OPEN ACCESS

Edited by:

Cecilia Gotor,

Institute of Plant Biochemistry and Photosynthesis, Spanish National Research Council (CSIC), Spain

Reviewed by:

Sung Chul Lee,

Chung-Ang University, South Korea Honghong $\mathrm{Hu}$,

Huazhong Agricultural University, China

*Correspondence: Bing-kai Hou bkhou@sdu.edu.cn

Specialty section:

This article was submitted to Plant Abiotic Stress,

a section of the journal

Frontiers in Plant Science

Received: 06 October 2021 Accepted: 29 November 2021 Published: 23 December 2021

Citation:

Liu Q, Dong G-r, Ma Y-q, Zhao S-m,

Liu X, Li X-k, Li Y-j and Hou B-k (2021)

Rice Glycosyltransferase Gene

UGT85E1 Is Involved in Drought

Stress Tolerance Through Enhancing

Abscisic Acid Response.

Front. Plant Sci. 12:790195.

doi: 10.3389/fpls.2021.790195

\section{Qian Liu, Guang-rui Dong, Yu-qing Ma, Shu-man Zhao, Xi Liu, Xing-kun Li, Yan-jie Li and Bing-kai Hou*}

The Key Laboratory of Plant Development and Environment Adaptation Biology, Ministry of Education, School of Life Science, Shandong University, Qingdao, China

Drought is one of the most important environmental constraints affecting plant growth and development and ultimately leads to yield loss. Uridine diphosphate (UDP)-dependent glycosyltransferases (UGTs) are believed to play key roles in coping with environmental stresses. In rice, it is estimated that there are more than 200 UGT genes. However, most of them have not been identified as their physiological significance. In this study, we reported the characterization of a putative glycosyltransferase gene UGT85E1 in rice. UGT85E1 gene is significantly upregulated by drought stress and abscisic acid (ABA) treatment. The overexpression of UGT85E1 led to an enhanced tolerance in transgenic rice plants to drought stress, while the ugt85e1 mutants of rice showed a more sensitive phenotype to drought stress. Further studies indicated that UGT85E1 overexpression induced ABA accumulation, stomatal closure, enhanced reactive oxygen species (ROS) scavenging capacity, increased proline and sugar contents, and upregulated expression of stress-related genes under drought stress conditions. Moreover, when UGT85E1 was ectopically overexpressed in Arabidopsis, the transgenic plants showed increased tolerance to drought as well as in rice. Our findings suggest that UGT85E1 plays an important role in mediating plant response to drought and oxidative stresses. This work may provide a promising candidate gene for cultivating drought-tolerant crops both in dicots and monocots.

Keywords: UGT85E1, glycosyltransferase, abscisic acid (ABA), reactive oxygen species (ROS), drought stress, rice

\section{INTRODUCTION}

Drought is one of the most important environmental cues that affect crop production. It is reported that drought stress will lead to a 50\% yield reduction in the reproductive stage of the crops (Hu and Xiong, 2014; Lesk et al., 2016). Therefore, improving the drought tolerance of crops is an urgent need when humans face more and more serious water shortages.

Abscisic acid (ABA) has long been considered the most important plant hormone involved in drought stress responses (Schachtman and Goodger, 2008). When plant roots sense a lack of soil water, they produce large amounts of ABA. The increase of ABA in plant cells activates intracellular signal conversion and protein phosphorylation and then regulates the specific binding process of transcription factors such as ABF (ABRE-binding factor) and AREB (ABA-responsive element binding protein), inducing the expression of downstream stress 
response genes, which is the main mechanisms of drought resistance in plants (Lee and Luan, 2012; Daszkowska-Golec and Szarejko, 2013; Ali et al., 2018), namely the so-called ABAdependent pathway. In addition, plants can also produce stress resistance through promoting root elongation, leaf curling, and helping plants maintain tissue water, namely the so-called ABA independent pathway (Hu and Xiong, 2014).

Under stress conditions, secondary metabolic regulation in plants is also an important way to cope with adverse environments, because the secondary metabolism of plants is the result of the plant interaction with the environments during plant evolution (Cook et al., 2004). For example, flavonoids, and anthocyanins increase in Arabidopsis when facing drought stress (Nakabayashi et al., 2014). UDP-Glycosyltransferases (UGTs) are a kind of enzyme that can catalyze the covalent addition of sugars to a broad range of secondary products and the UGT superfamily is believed to play crucial roles in modulating secondary metabolic balance in plant cells (Lim and Bowles, 2004; Bowles et al., 2005). Therefore, it would be very important to explore the role of UGTs in regulating metabolism-related stress responses. A previous study reported that UGT79B2 and UGT79B3 can glycosylate anthocyanins, and the overexpression lines accumulate more anthocyanins to adapt to drought stress (Li et al., 2017). Similarly, maize glycosyltransferase UFGT2 modifies flavonols and contributes to plant acclimation to abiotic stresses (Li et al., 2018). A rice glycosyltransferase UGT83A1 glycosylates most of the lignin precursors and flavonoids, its overexpressing lines showed strong resistance to salt, drought, and cold stress (Dong et al., 2020). As important signaling molecules, phytohormones are also regulated by molecular glycosylation to address the stress responses (Bowles et al., 2006; Wong et al., 2006). For example, UGT71C5 and UGT75B1 are UGT genes that target ABA in Arabidopsis. Their knockout mutants in the seedling stage showed higher resistance than the wild type to salt stress (Liu et al., 2015; Chen et al., 2020). Glycosylation of auxin also affects plant stress responses. UGT74E2-mediated glycosylation of IBA enhances drought stress resistance in Arabidopsis (Tognetti et al., 2010). Secondary metabolism-linked UGTs are indispensable in regulating plant stress responses, although most current studies in this aspect are centered on the model plant Arabidopsis.

In rice, it is estimated that there would be more than 200 UGT genes. However, most of them have not been identified as their physiological significance. In this study, we reported the characterization of a rice glycosyltransferase gene, UGT85E1. The UGT85E1 gene is significantly upregulated by drought stress and ABA treatments. The overexpression of UGT85E1 led to the enhanced tolerance in transgenic rice plants to drought stress, while the rice $u g t 85 e 1$ mutants showed the more sensitive phenotype to drought stress. UGT85E1 overexpression induced stomatal closure, ABA accumulation, and enhanced reactive oxygen species (ROS) scavenging capacity under drought stress conditions. When UGT85E1 was ectopically overexpressed in Arabidopsis, the transgenic lines showed increased tolerance to drought conditions as well as in rice. Our findings suggest that glycosyltransferase gene UGT85E1 plays an important role in mediating plant response to drought and oxidative stresses. This work may provide a promising candidate gene for cultivating drought-tolerant crops both in dicots and monocots.

\section{RESULTS}

\section{UGT85E1 Gene Is Induced by Mannitol and ABA}

In our attempt to screen rice UGT genes responsive to abiotic stress, UGT85E1 (LOC_Os02g51900) drew our attention because of its response to drought stress. We examined the responsiveness of UGT85E1 to mannitol and exogenous ABA via real-time quantitative reverse transcription PCR (qRT-PCR). The expression level of UGT85E1 under $200 \mathrm{mM}$ mannitol treatment gradually increased from 6 to $24 \mathrm{~h}$, decreasing slightly at $48 \mathrm{~h}$. The induction of UGT85E1 was also observed from 6 to $48 \mathrm{~h}$ under $100 \mu \mathrm{M}$ ABA treatment (Figure 1A). These two treatments significantly induced the expression of UGT85E1, suggesting that this gene may be involved in drought stress. To analyze the function of UGT85E1, we created the overexpression lines and mutant lines of the UGT85E1 gene. Two overexpression lines (OE-3 and OE-5) and two CRISPR/cas9 mutant lines (ko-8 and ko-9) were subjected to drought stress for further analysis (Figure 1B, Supplementary Figure 1).

\section{The Phenotype of UGT85E1 Transgenic Plants Under Drought Stress}

Under normal conditions, 2-week-old UGT85E1 overexpression lines and mutants showed normal and similar growth phenotypes with wild-type plants (WT). After $150 \mathrm{mM}$ mannitol treatment, most mutants appeared more withered than the WT, however, the two overexpression lines performed better growth than WT (Figures 2A,C). Survival rates showed consistency with the phenotype observation (Figures 2B,D). We also conducted drought treatments using 4-week-old mutants and overexpression lines. No growth difference of mutants and overexpression lines when compared with WT can be observed under soil culture and normal irrigation conditions. After deprival of irrigation for about 1.5 weeks, some mutant plants wilted. After re-irrigating for 2 days, the mutants were more withered and showed a lower survival rate than that of WT (Figures 2E,F). However, the overexpression lines grew better than that of WT under drought conditions, showing more green, upright petiole, and a much higher survival rate (Figures 2G,H). These experiments proved that UGT85E1 is involved in drought stress response and its overexpression can significantly enhance the drought tolerance of rice.

\section{UGT85E1 Is Involved in the Modulation of Endogenous ABA Level and Stomatal Aperture}

Upon exposure to drought stress, water loss of plant leaves mainly occurred through the stomatal opening. Thus, we investigated the stomatal aperture of 4-week-old different transgenic lines under dehydration conditions. Here, the stomatal opening was classified into three types, i.e., fully open, partially open, and completely closed as shown in Figure 3A. Before dehydration, 

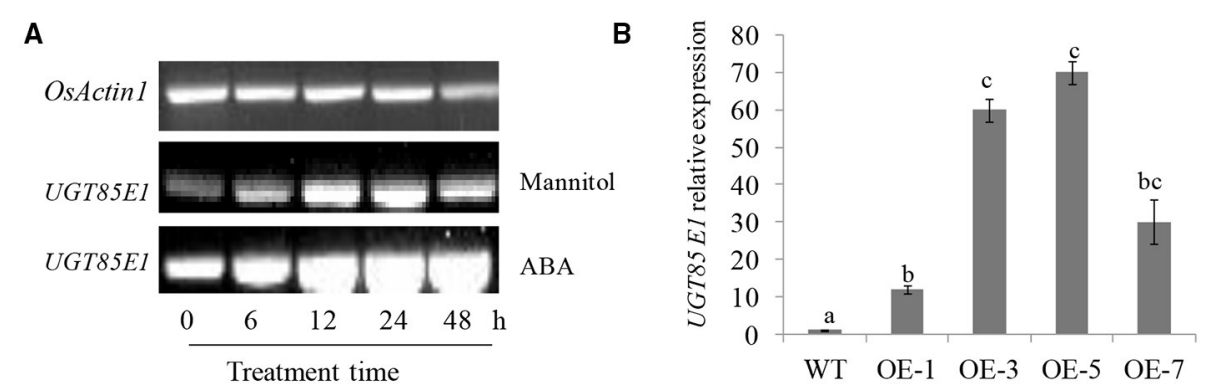

FIGURE 1 | The induced expression of UGT85E1 and the preparation of UGT85E1 transgenic plants. (A) UGT85E1 transcript level was up-regulated by 200 mM Mannitol and $100 \mu \mathrm{M}$ ABA treatments through reverse transcription-PCR (RT-PCR) analysis; (B) Generation and identification of UGT85E1 overexpression lines in rice. OsActin1 was used as the internal control. Error bars indicate the SD of three independent experiments. Statistically significant differences were determined by one-way ANOVA, followed by Tukey's test $(P<0.05)$.

the ratio of three types of stomatal opening is roughly the same between WT, mutants, and overexpression lines. After $2 \mathrm{~h}$ of dehydration of detached leaves, however, the proportion of stomatal closure increased and the proportion of partially open stomata significantly decreased in overexpression line OE-3 compared with WT (Figure 3B). The mutant line ko-8 was on the contrary with less closed and more open stomata compared to WT. For example, the percentage of completely closed stomata in WT, ko-8, and OE-3 lines was 30.1, 17.3, and $70 \%$, respectively (Figure 3B). This result showed that the drought tolerance of UGT85E1 transgenic rice was due to the reduced stomatal opening.

Then, we detected the rate of water loss from detached leaves of each UGT85E1 transgenic line within $120 \mathrm{~min}$. The result indicated that the two overexpression lines exhibited lower water loss than that of WT, but the mutants showed more water loss (Figure 3C).

It is well-known that $\mathrm{ABA}$ can induce stomatal closure. We thus examined the endogenous ABA levels in UGT85E1 transgenic lines and WT under normal and drought stress conditions. Although ABA levels were similar for different genotypic plants under control conditions, overexpression lines showed much higher ABA levels than WT under drought stress, whereas the mutants showed lower ABA levels (Figure 3D). This result prompted us to hypothesize that UGT85E1 may be involved in regulating $\mathrm{ABA}$ biosynthesis in drought stress. To confirm this, the transcript levels of ABA biosynthesis and responsive genes were analyzed. As shown in Figure 4A, the expression levels of ABA biosynthesis genes OsABA1, $O s A B A 2, O s A B A 4$, and OsNCED1 and ABA-responsive genes $O s A B I 5$ and OsbZIP23 were highly upregulated by drought stress in the UGT85E1 overexpression lines as compared with WT, while in the mutants, they were down-regulated. The upregulation or downregulation of ABA biosynthesis and responsive genes would change the sensitivity of seed germination to exogenously applied ABA. As shown in Figures $4 B, C$, our investigation indicated that the root length of overexpression lines was shorter than WT seedling under 5$10 \mu \mathrm{M}$ of ABA treatment, whereas the mutants showed the longer root. These results suggest that the role of UGT85E1 in enhancing drought tolerance may be due to the enhanced ABA response in rice.

\section{Overexpression of UGT85E1 Enhances ROS Scavenging in Transgenic Plants}

Stresses often lead to the accumulation of hydrogen peroxide $\left(\mathrm{H}_{2} \mathrm{O}_{2}\right)$ in plant cells, and the ability of cells to remove $\mathrm{H}_{2} \mathrm{O}_{2}$ reflects the level of resistance to stresses (Li et al., 2004). It was reported that $\mathrm{ABA}$ could significantly promote the activities of ROS scavenging enzymes such as SOD (Superoxide dismutase), APX (Ascorbate peroxidase), and CAT (Catalase) under stress conditions (Jiang and Zhang, 2002; Xu et al., 2018). Given that UGT85E1 is involved in ABA response as mentioned above, we thus examined ROS accumulation and the relative expression of several genes encoding ROS scavenging enzymes in UGT85E1 overexpression lines and mutants. $3,3^{\prime}$-Diaminobenzidine (DAB) staining indicated that $\mathrm{H}_{2} \mathrm{O}_{2}$ accumulated more in ugt85e1 mutants than WT, the overexpression lines showed the opposite changes under mannitol treatment (Figure 5A). Transcript levels of several genes encoding ROS scavenging enzymes were significantly upregulated in overexpression lines, but downregulated in ugt85e1 mutant lines compared with WT after $250 \mathrm{mM}$ mannitol treatment (Figure 5B). These results indicated that UGT85E1 overexpression promotes the scavenging of ROS.

As osmoprotective molecules, proline and sugar are recognized to protect cells against osmotic stress. Numerous studies have demonstrated that the proline and sugar accumulation is enhanced in response to different abiotic stresses and also by the induction of ABA (Verslues et al., 2007; Szabados and Savoure, 2010; Vishwakarma et al., 2017; Živanović et al., 2020). Here, we measured the contents of proline and soluble sugar in the UGT85E1 overexpression lines and mutants subjected to drought treatment. Our results showed that much more proline and soluble sugar were accumulated in OE-3 and OE-5 plants, but less in ko-8 and ko-9 compared with WT (Figures 5C,D).

We also investigated the expression changes of four stressrelated genes (OsRAB16, OsRAB21, OsDREB2B, and OsDREB1A) after $250 \mathrm{mM}$ mannitol treatment. The results showed that the 
A

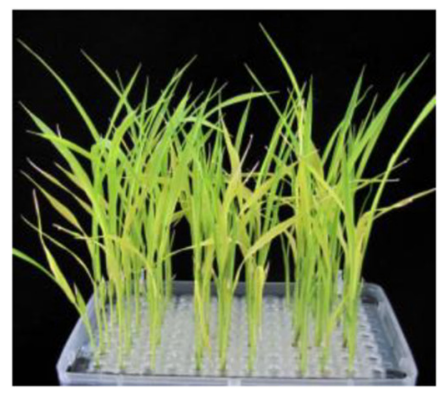

$$
\begin{array}{lcc}
\text { WT } & \text { ko-8 } & \text { ko-9 } \\
\text { Control }
\end{array}
$$
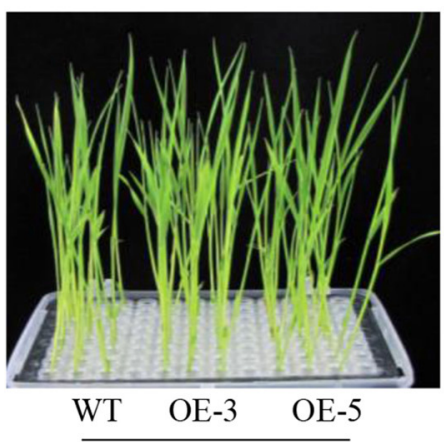

Control

E

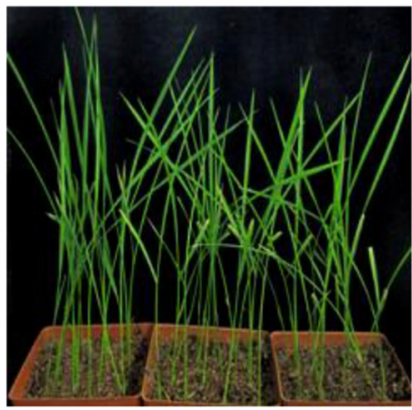

$$
\text { WT ko-8 ko-9 }
$$

G

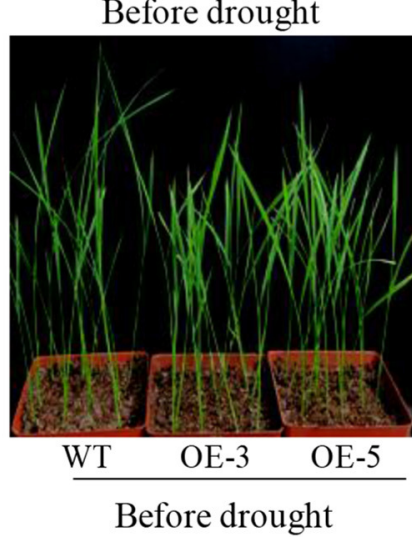

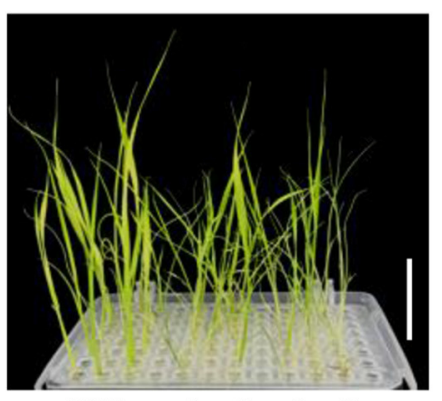

WT ko-8 ko-9
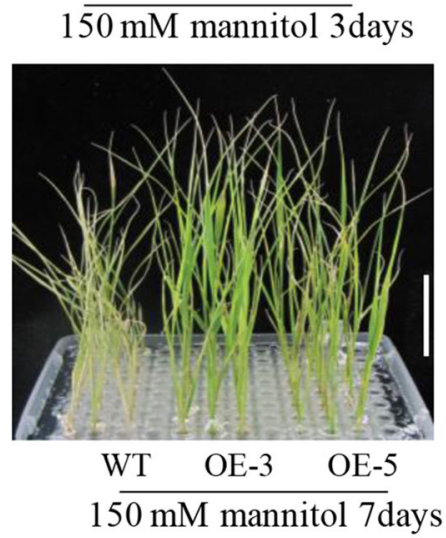

D
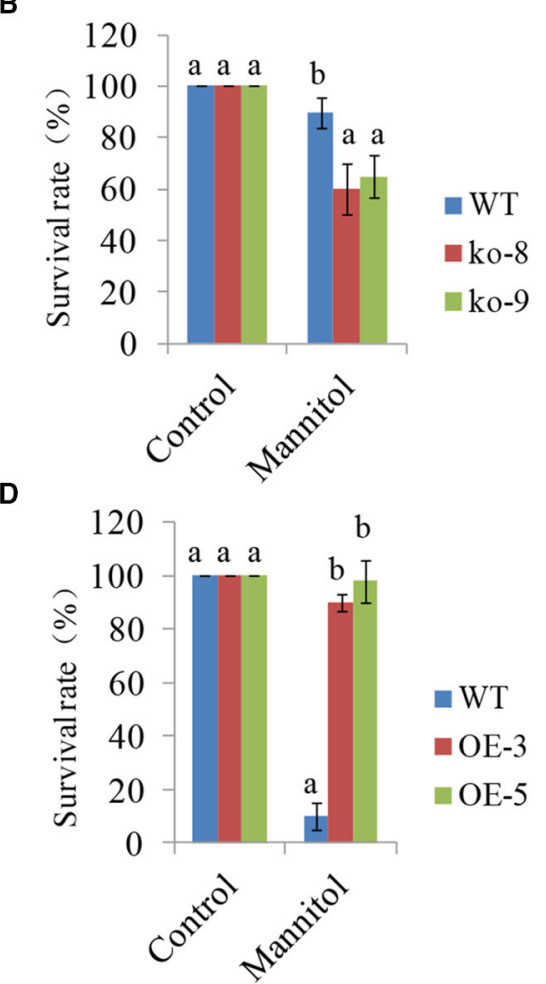

$\mathbf{F}$

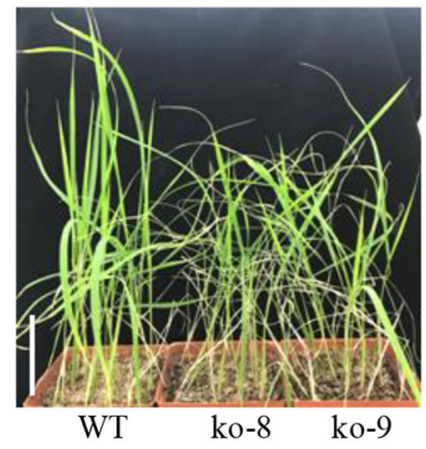

Drought 6 days and recovery 2 days $\mathbf{H}$

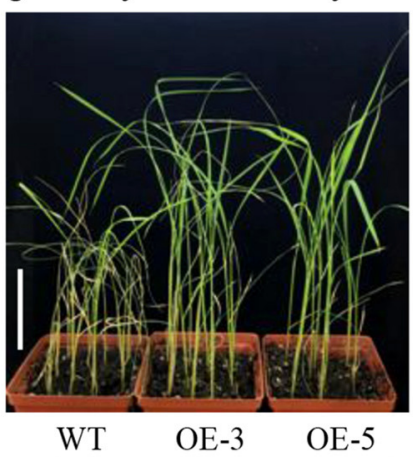

Drought 10 days and recovery 2 days

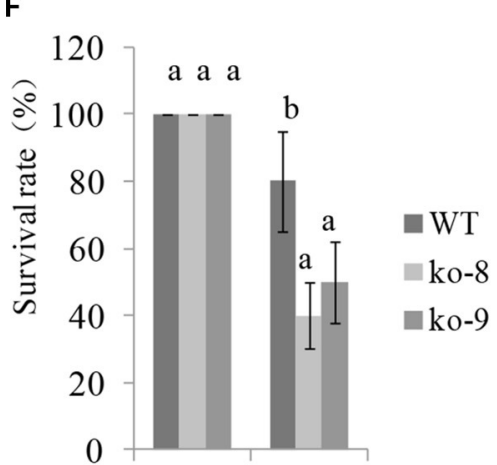

Before After

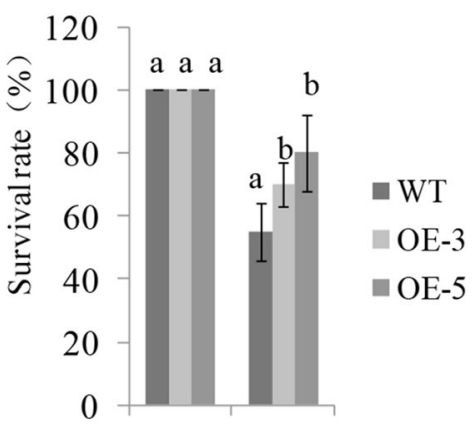

Before After

FIGURE 2 | Growth phenotypes of UGT85E1 overexpression lines and mutants under mannitol and drought treatments. (A,C) Phenotypes of UGT85E1 mutants and overexpression lines after $150 \mathrm{mM}$ Mannitol treatment, scale bar = $5 \mathrm{~cm}$; (B,D) The survival rate of mutant lines and overexpression lines after $150 \mathrm{mM}$ Mannitol treatment; (E,G) Phenotypes of UGT85E1 mutants and overexpression lines were grown in the soil after drought treatment for 6-10 days and recovery for 2 days, scale bar $=8 \mathrm{~cm} ; \mathbf{( F , H )}$ The survival rate of mutants and overexpression lines after drought treatment. Error bars indicate the SD of three independent experiments. Statistically significant differences were determined by one-way ANOVA, followed by Tukey's test $(P<0.05)$. 


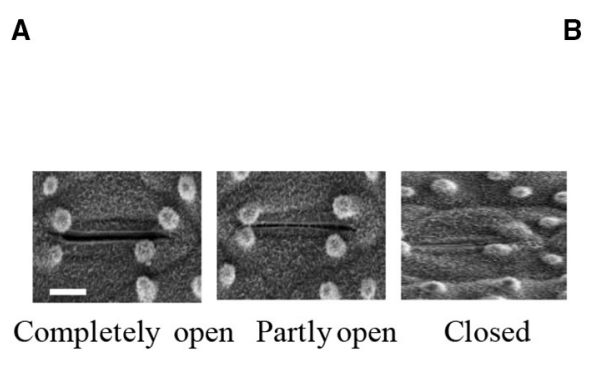

Completely open Partlyopen
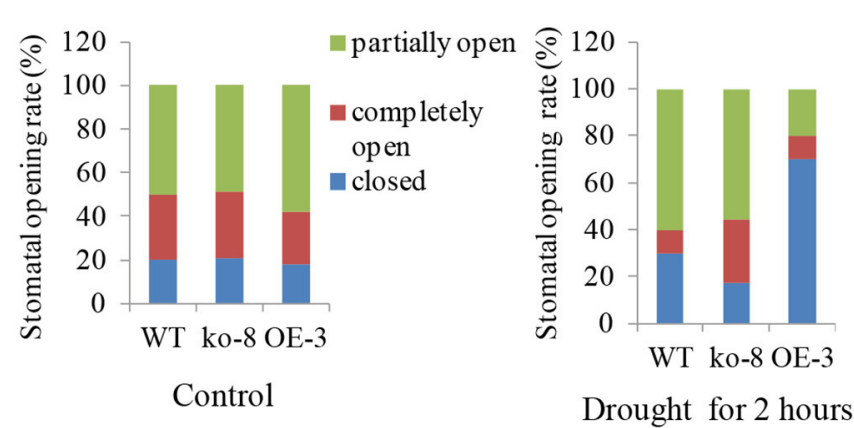

Drought for 2 hours

c

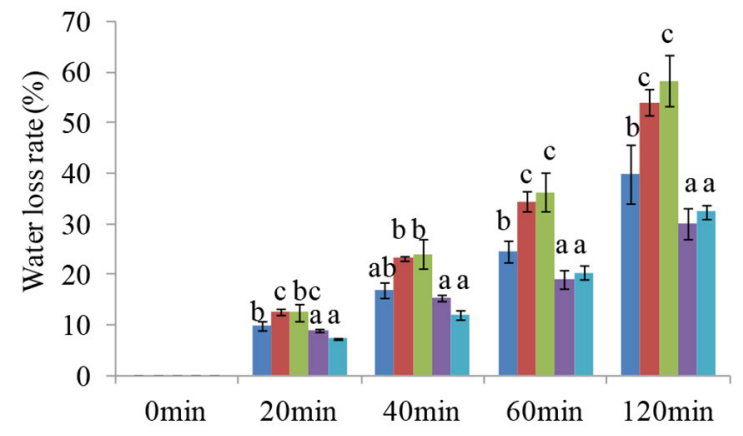

D

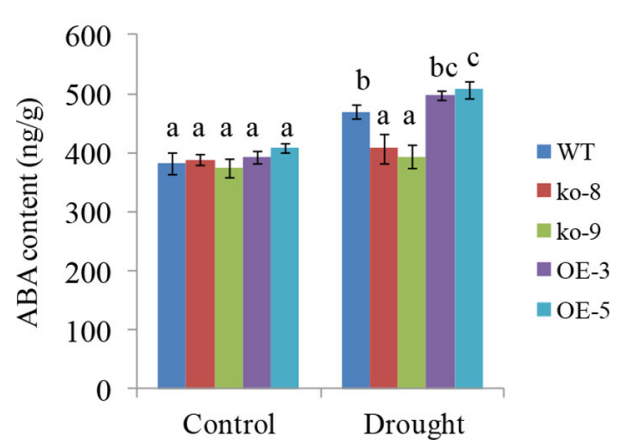

FIGURE 3 | Stomatal aperture, Water loss rates, and abscisic acid (ABA) levels in UGT85E1 transgenic plants under drought treatment. (A) A scanning electron microscope was used to observe stomatal opening. Bar $=5 \mu \mathrm{m}$; (B) The ratio of stomatal opening and closing of WT and UGT85E1 transgenic lines before and after drought. (C) Water loss rates of detached UGT85E1 transgenic leaves within 120 min. (D) Detection of ABA content in WT and UGT85E1 transgenic lines under normal and drought stress conditions. Error bars indicate the SD of three independent experiments. Statistically significant differences were determined by one-way ANOVA, followed by Tukey's test $(P<0.05)$.

expression levels of four genes in overexpression lines were significantly higher than that in WT, their expression levels in mutant lines were lower than that in WT (Figure 5E). The above-mentioned results suggest that the UGT85E1 gene exerts its important influence on these stress-related genes, directly or indirectly, and plays a broad role when facing stress conditions.

\section{UGT85E1 Can Enhance Oxidative Stress Tolerance in Detached Rice Leaves}

To further investigate the role of UGT85E1 in scavenging ROS, we also tested the detached leaves of the overexpression lines and mutant lines with the exogenously applied $\mathrm{H}_{2} \mathrm{O}_{2}$ and herbicide methyl viologen (MV), which can generate ROS locally in chloroplasts. As shown in Figure 6A, under the control condition, there was no difference in the green color of the detached leaves from each of the lines soaked in distilled water for 1 week. When treated with $2 \% \mathrm{H}_{2} \mathrm{O}_{2}$ or $10 \mu \mathrm{M} \mathrm{MV}$, we can see that most overexpression samples were greener than WT, and mutant samples were browner than WT. The determination of chlorophyll contents also confirmed this observation (Figure 6B). This result again demonstrated that the overexpression of UGT85E1 can enhance the ability to scavenge $\mathrm{H}_{2} \mathrm{O}_{2}$ and to resist oxidative stress.

\section{Ectopic Expression UGT85E1 in Arabidopsis Significantly Enhances Drought Stress Tolerance}

To further verify the effect of UGT85E1 in enhancing drought stress tolerance, we overexpressed UGT85E1 in Arabidopsis and investigated the drought tolerance using two overexpression lines OE-4 and OE-7 (Figure 7A). Dehydration assay indicated that Arabidopsis OE-4 and OE-7 had a lower water loss rate than WT within 120 min (Figure 7B). When 3-week-old soilgrown seedlings were subjected to drought stress for 1 week and followed by re-irrigation for $24 \mathrm{~h}$, we can see that the overexpression lines grew better than WT. Not only the leaves of OE-4 and OE-7 were fully extended but almost all survive. However, about half of the WT wilted (Figures 7B,C,D). These results suggest that UGT85E1 overexpression in Arabidopsis also contributes to drought tolerance.

We also investigated the ROS levels in Arabidopsis overexpression lines and WT plants under stress conditions. The DAB and nitroblue tetrazolium test (NBT) staining indicated that ROS accumulated much less in UGT85E1 transgenic Arabidopsis lines than in WT (Figures 8A,B). We further analyzed the expression of the abiotic stress-related genes and found that the transcript levels of AtRD29A, AtRD29B, AtDREB1A, and AtDREB1B in transgenic 

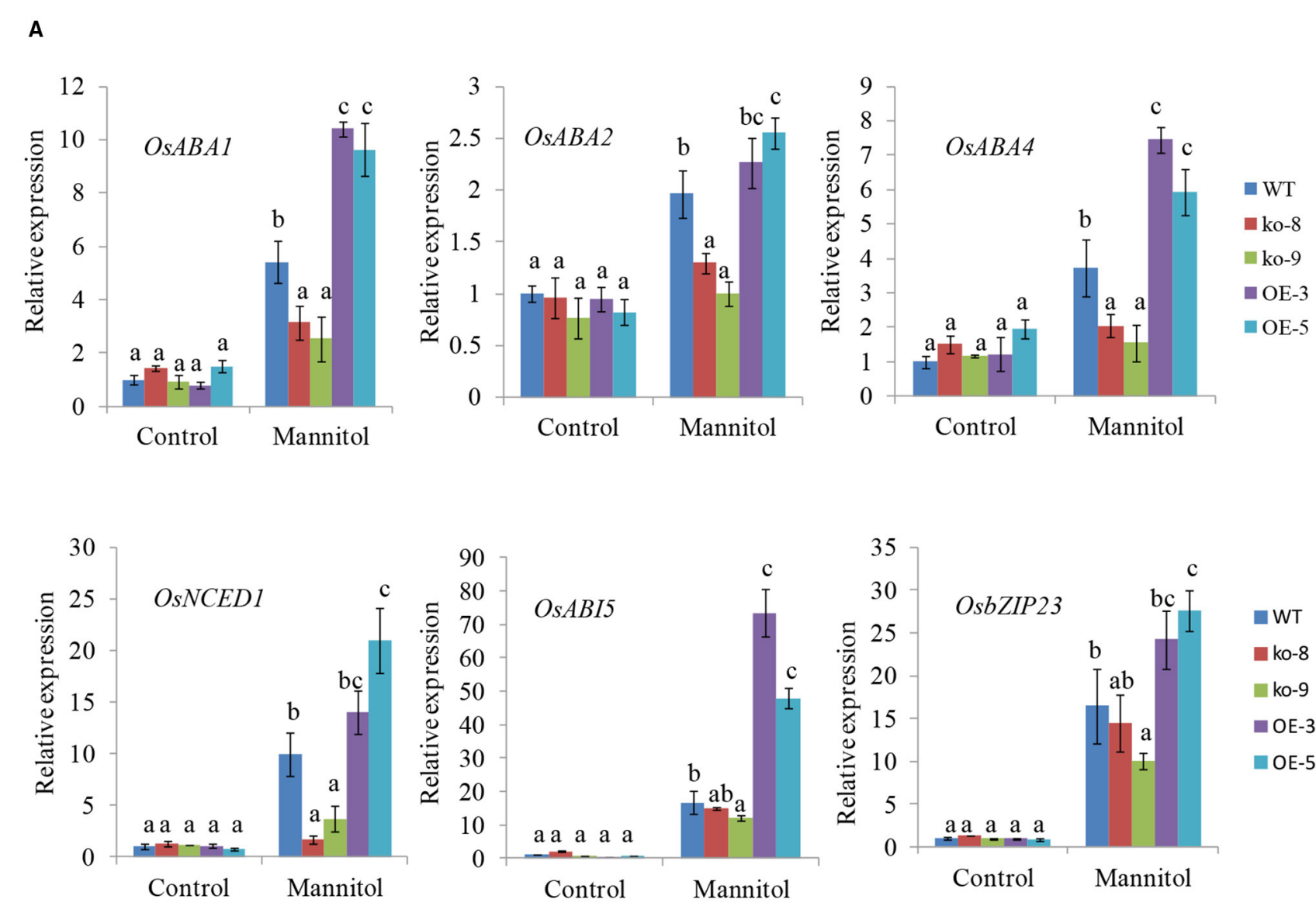

B

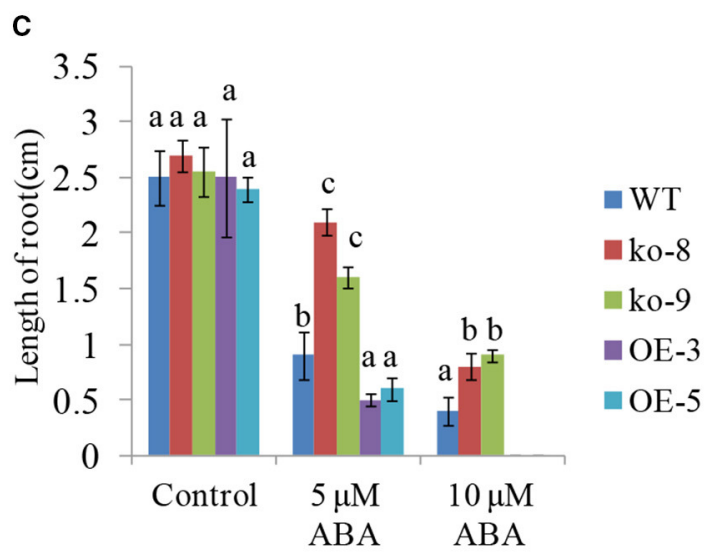

FIGURE 4 | Expressions of ABA-related genes and ABA responses in UGT85E1 transgenic plants under normal and treatment conditions. (A) Real-time PCR analysis of the expression of ABA biosynthesis and responsive genes under normal and mannitol treatment conditions; (B) Influence of ABA treatments on post-germination growth of UGT85E1 transgenic rice seedlings; (C) Rice root lengths of UGT85E1 transgenic lines were recorded under ABA treatments and control condition. Bars = $0.5 \mathrm{~cm}$. Error bars indicate the SD of three independent experiments. Statistically significant differences were determined by one-way ANOVA, followed by Tukey's test $(P<0.05)$.

Arabidopsis lines were higher than in WT (Figure 8C). These ectopic expression data provide more powerful evidence for the involvement of UGT85E1 in enhancing drought stress tolerance.

\section{DISCUSSION}

Rice is the most important food in the world that feeds more than half of the world's population. However, with the deterioration 
A

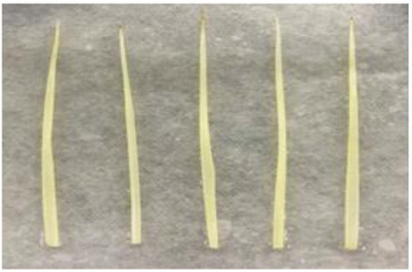

WT ko-8 ko-9 OE-3 OE-5

Control

B
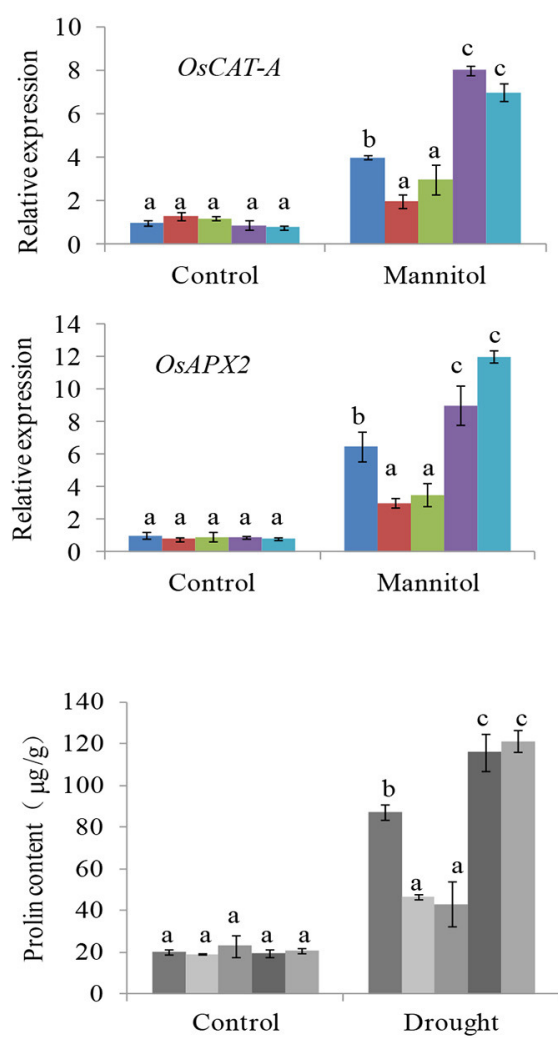

E
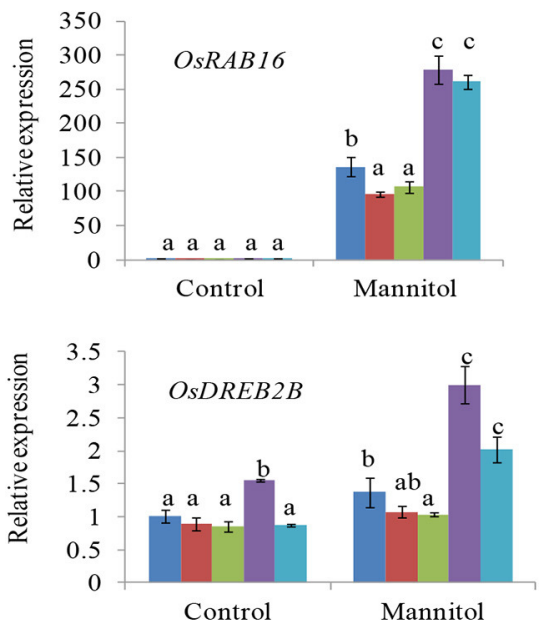

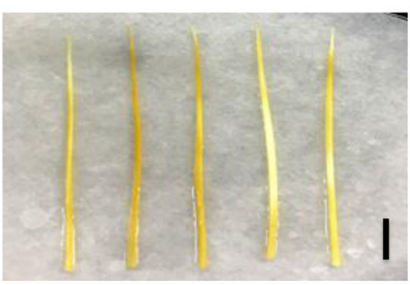

WT ko-8 ko-9 OE-3 OE-5 250 mMMannitol $24 \mathrm{~h}$
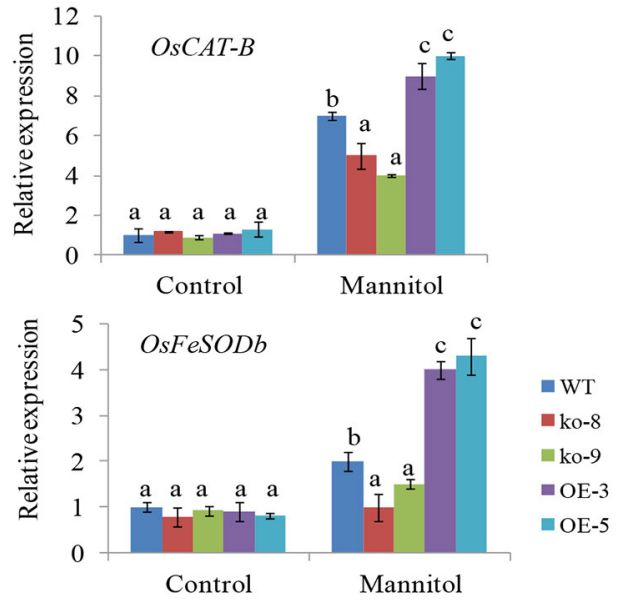

D
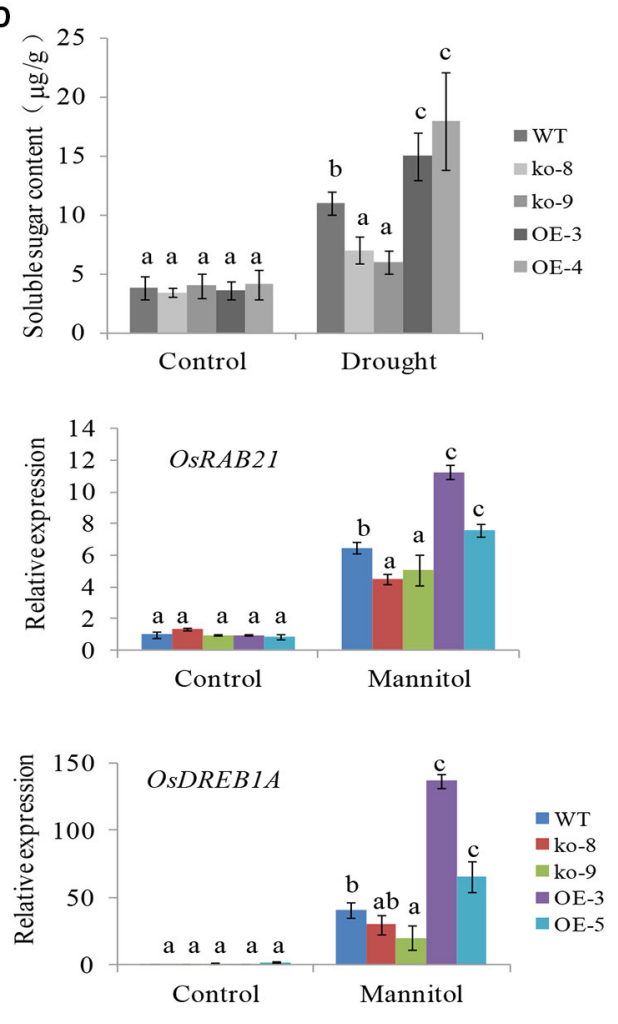

FIGURE 5 | Accumulation of hydrogen peroxide $\left(\mathrm{H}_{2} \mathrm{O}_{2}\right.$ ) and the expression levels of reactive oxygen species (ROS) scavenging genes and drought stress response genes in UGT85E1 transgenic lines. (A) DAB staining of UGT85E1 transgenic lines under control and mannitol treatments, scale bar $=2 \mathrm{~cm}$; (B) Transcription levels of ROS scavenging enzyme genes in UGT85E1 transgenic lines under normal and drought stress conditions; (C,D) Proline and soluble sugar contents in rice leaf tissues 
FIGURE 5 | sampled from UGT85E1 transgenic lines and WT plants under control and drought treatment conditions. (E) Expression levels of drought stress response genes in UGT85E1 transgenic lines. Error bars indicate the SD of three independent experiments. Statistically significant differences were determined by one-way ANOVA, followed by Tukey's test $(P<0.05)$.
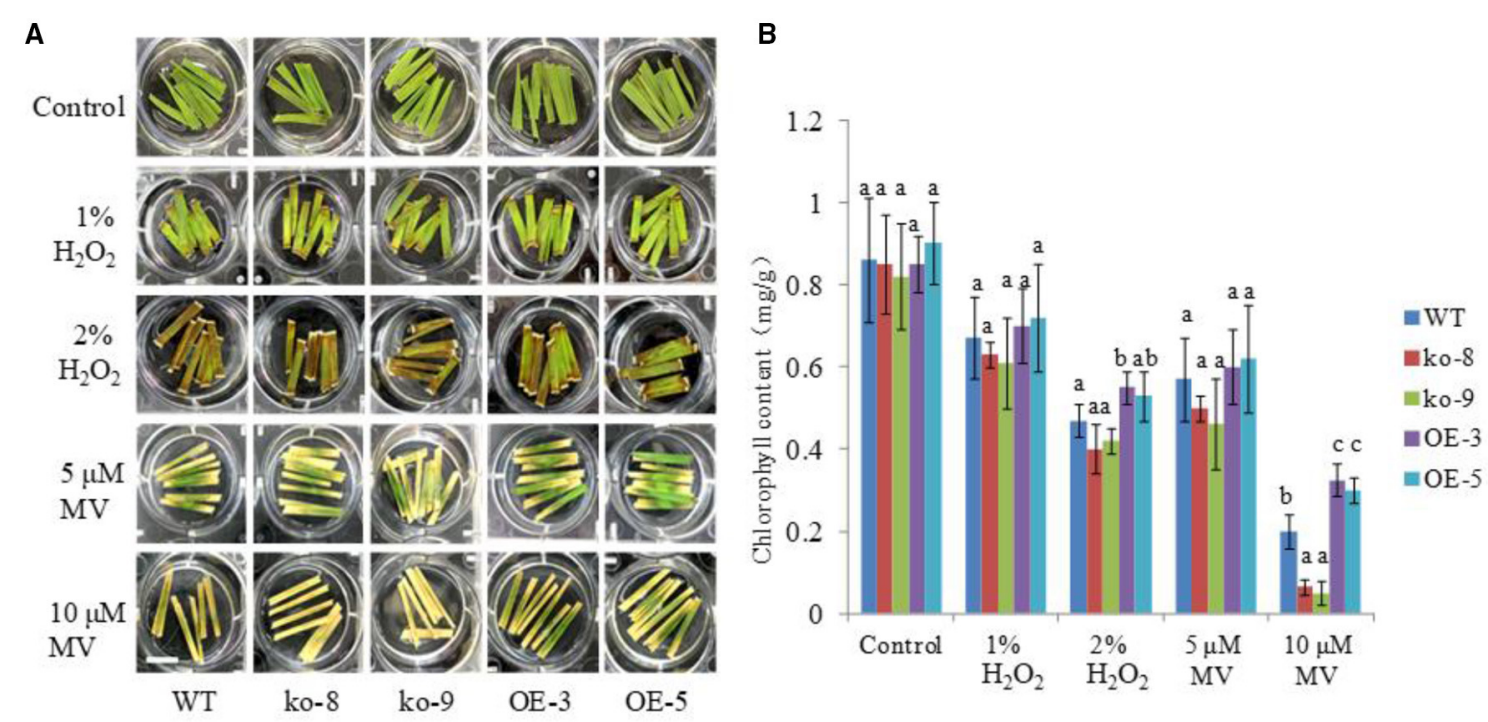

FIGURE 6 | Effects of $\mathrm{H}_{2} \mathrm{O}_{2}$ and methyl viologen (MV) treatments on the detached leaves of UGT85E1 transgenic lines. (A) Effects of different concentrations of $\mathrm{H}_{2} \mathrm{O}_{2}$ and MV on the leaves of UGT85E1 transgenic lines, bar $=2 \mathrm{~cm}$; (B) Determination of the chlorophyll contents of WT and UGT85E1 transgenic lines after $\mathrm{H}_{2} \mathrm{O}_{2}$ and MV treatments. Error bars indicate the SD of three independent experiments. Statistically significant differences were determined by one-way ANOVA, followed by Tukey's test $(P<0.05)$.

of the current global environment and uneven precipitation, the area of soil drying increased gradually. To meet the demand for food, cultivating rice varieties with drought tolerance would be very important. In this study, we characterized the UGT85E1 gene, a putative UGT gene that is involved in drought response. The transcript level of UGT85E1 was strongly induced by mannitol and ABA treatments. Its overexpression lines showed an enhanced survival rate and improved growth performance under mannitol treatment or drought treatment conditions. When it was introduced into Arabidopsis, the ectopically expressed UGT85E1 substantially enhanced drought tolerance. These findings suggest the UGT85E1 has promising application in the genetic improvement of crops with abiotic stress resistance.

Abscisic acid (ABA) is a key regulator of both plant development and stress response, including seed germination and dormancy, root development, stomatal movement, and abiotic stress tolerance (Jeon et al., 2010; Watkins et al., 2017). Following these above findings, UGT85E1 overexpression plants had the increased ABA level with enhanced drought tolerance, more closed stomata, and less water loss rate than that in WT plants when confronting drought stress. ROS at the physiological level is believed to act as a signaling molecule that regulates plant adaptation to various stresses, while ROS at an excessive level often causes significant damage to plant cells, leading to deleterious effects on stress tolerance (Choudhury et al., 2013; Xie et al., 2014; You and Chan, 2015; Tognetti et al., 2017). ABA plays a crucial role in ROS homeostasis and it can regulate the expression of ROS producing and scavenging genes. For example, abiotic stress-induced ABA accumulation triggers the increased generation of ROS and upregulates the activities of antioxidant enzymes in maize leaves (Jiang and Zhang, 2002). Arabidopsis glutathione peroxidase genes are also regulated by abiotic stresses together with ABA (Milla et al., 2003). Antioxidant enzymes were often involved in the stress responses of plants. For instance, the ascorbate peroxidase gene OsAPX2 plays a positive role in chilling tolerance by enhancing $\mathrm{H}_{2} \mathrm{O}_{2}$ scavenging (Zhang et al., 2013). OsCATB prevents the excessive accumulation of $\mathrm{H}_{2} \mathrm{O}_{2}$ under water stress (Ye et al., 2011). Our results showed that the expression levels of several ROS scavenging-related genes clearly increased in the UGT85E1 overexpression plants and decreased in mutant lines. Consistent with this, the UGT85E1 overexpression lines exhibited better growth under oxidative stress caused by the direct application of $\mathrm{H}_{2} \mathrm{O}_{2}$ or $\mathrm{MV}$ treatment. In contrast, mutant plants showed enhanced sensitivity to oxidative stress. These results suggest that the improved drought tolerance of UGT85E1 overexpression plants may be due to the ABA-dependent ROS scavenging. In addition, more proline and soluble sugar as osmotic protective compounds were also accumulated in UGT85E1 overexpression plants. Previous reports demonstrated that salinity-induced proline accumulation is dependent on ABA, which is also consistent with the proposal that proline is associated with redox regulation and might serve as an antioxidant (Van Breusegem and Dat, 2006; Hoque et al., 2008). All of these observations 
A

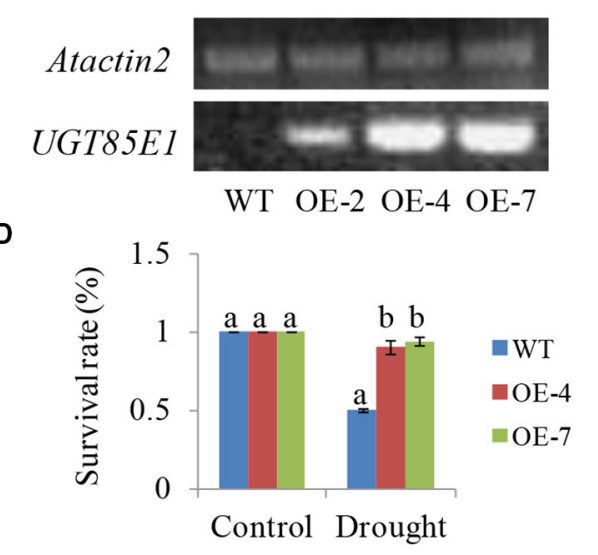

B

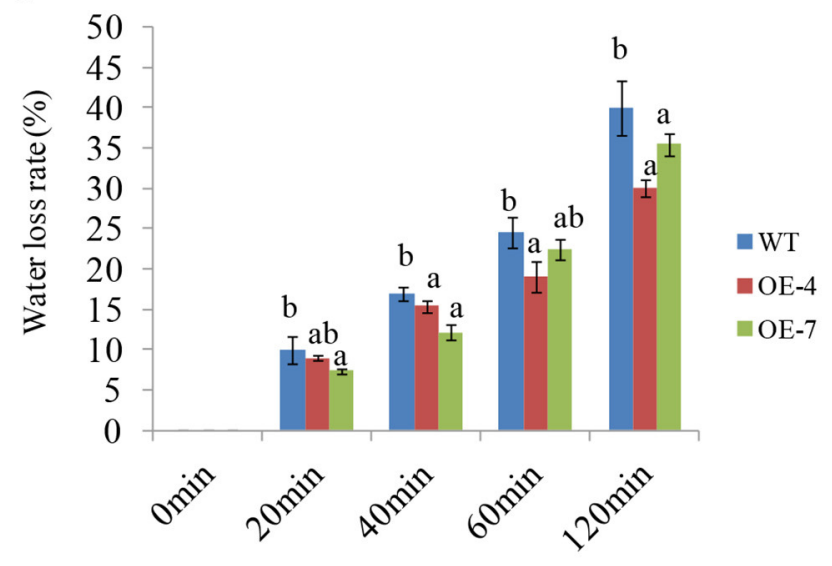

C

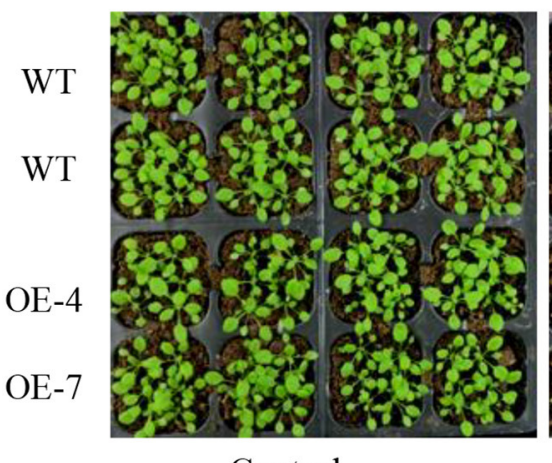

Control

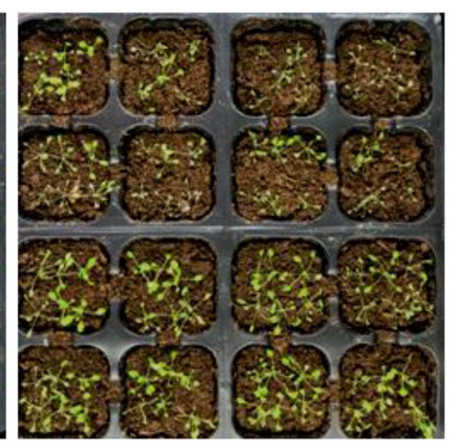

Drought 6days

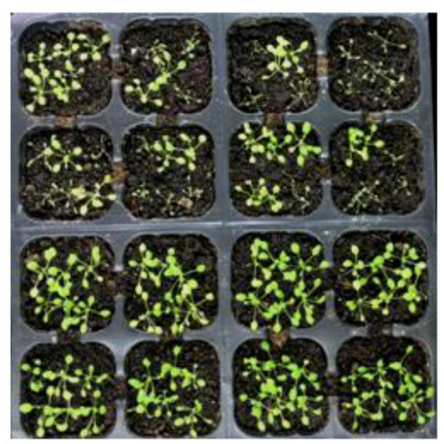

Recovery one day

FIGURE 7 | Drought-tolerant phenotype of UGT85E1 transgenic Arabidopsis. (A) Identification of overexpressing UGT85E1 transgenic Arabidopsis lines through RT-PCR, the Atactin2 was used as the internal control; (B) Detection of water loss rates of detached leaves from UGT85E1 transgenic Arabidopsis lines within 120 min; (C) Growth phenotype UGT85E1 transgenic lines after drought treatment for 6 days and recovery for 1 day with water; Untreated plants were used as the control; (D) Survival rates of UGT85E1 transgenic Arabidopsis lines and wild-type WT plants after drought treatment were examined. Error bars indicate the SD of three independent experiments. Statistically significant differences were determined by one-way ANOVA, followed by Tukey's test $(P<0.05)$.

highlight a link between the altered $\mathrm{ABA}$ responses and the changed $\mathrm{H}_{2} \mathrm{O}_{2}$ levels in UGT85E1 transgenic plants.

Moreover, UGT85E1 gene expression also promotes the upregulation of multiple stress-related genes in rice and Arabidopsis under drought conditions, including ABA biosynthesis and signaling genes. But the molecular mechanism of UGT85E1 involved in drought tolerance is not known. Because UGT85E1 is a putative UDP-dependent glycosyltransferase, which mainly catalyzes the glycosylation of plant secondary products, its influence on the expression levels of ABA biosynthesis and signaling genes would depend on its biochemical function. Unfortunately, the relevant substrate(s) has not been identified yet in this study, although we examined a lot of secondary plant metabolites. Thus, the precise mechanism for how UGT85E1 functions is not known at the moment. Here, we can suppose several possible action modes of UGT85E1. The first possibility is that UGT85E1 glycosylates ABA-antagonistic factors such as cytokinins. Several studies have revealed the interplay between cytokinins and ABA, which act antagonistically in regulating stress responses. For instance, the trans-zeatin riboside decreases significantly in the sunflower plants, while the endogenous ABA content was increased when suffering from drought stress (Hansen and Dörffling, 2003). AHK2, AHK3, and A-type ARRs act as negative regulators in the cold stress signaling pathways via inhibiting ABA response (Jeon et al., 2010). It is also reported that cytokinin-deficient ipt1357 mutant and CKX overexpression lines are more sensitive to ABA compared with the wild type, leading to a higher induction of ABA signaling genes by stresses and the enhanced stress tolerance (Nishiyama et al., 2011). A comparative genome-wide analysis of the leaves of arr1,10,12 and WT plants under dehydration conditions suggested a cytokinin signaling-mediated network controlling plant adaptation to drought via many ABA-responsive genes (Nguyen et al., 2016). If UGT85E1 glycosylates some unidentified cytokinin(s), the removal of the antagonistic effect would promote the expression levels of ABA biosynthetic and signaling genes, leading to enhanced drought tolerance. The second possibility is that UGT85E1 glycosylates some inhibitor(s) of some transcription factors and relieves the inhibition effect on the transcription of ABA biosynthetic and signaling genes. In addition, we can also not exclude the possibility that UGT85E1 may glycosylate the 
A

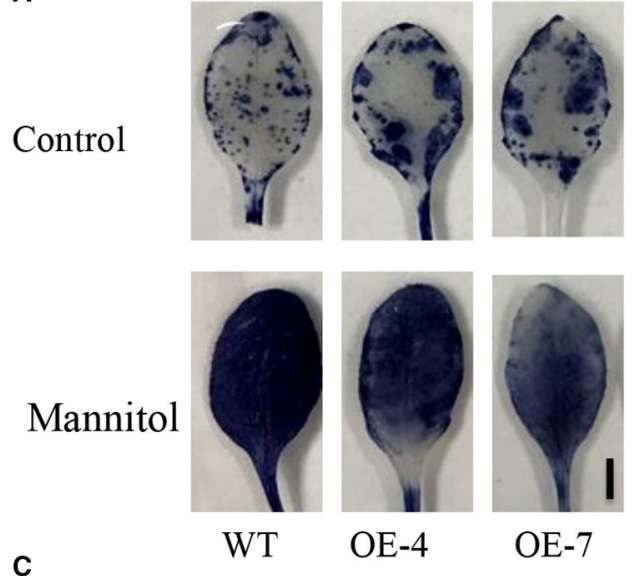

C

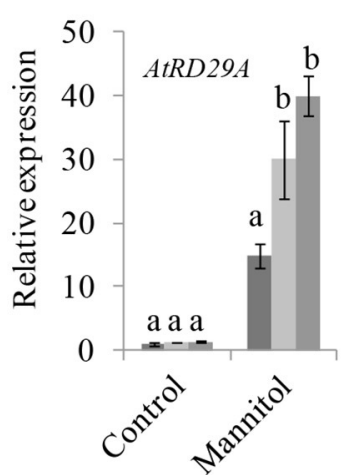

B

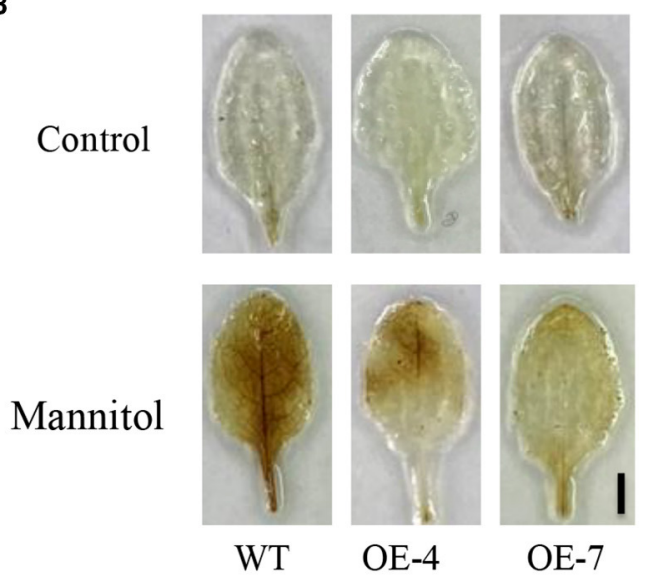

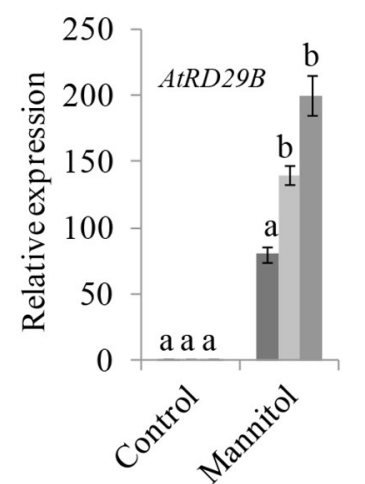
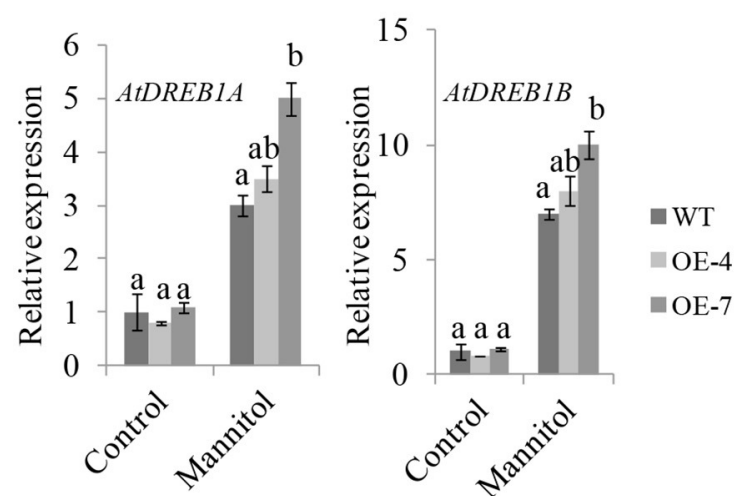

FIGURE 8 | Overexpression of UGT85E1 enhanced antioxidant ability of Arabidopsis plants. (A,B) Antioxidant capacity UGT85E1 transgenic lines assessed by nitroblue tetrazolium test (NBT) (A) and 3,3' -Diaminobenzidine (DAB) (B) staining after $250 \mathrm{mM}$ mannitol treatment for $12 \mathrm{~h}$, untreated plants were used as the control. (C) Expression levels of stress response genes were analyzed by Real-time qRT-PCR for UGT85E1 transgenic Arabidopsis lines under Mannitol treatment. Error bars indicate the SD of three independent experiments. Statistically significant differences were determined by one-way ANOVA, followed by Tukey's test $(P<0.05)$.

metabolites of $\mathrm{ABA}$ and regulate $\mathrm{ABA}$ synthesis by a feedback mechanism, just like the case of SA synthesis regulation by UGT76D1 (Huang et al., 2018). It is estimated that there are more than 200 UGTs in rice (Caputi et al., 2012). UGT85E1 is close to the UGT85 family in Arabidopsis. It is reported that UGT85A1 is responsible for glycosylating trans-zeatin which is a kind of cytokinin (Hou et al., 2004; Jin et al., 2013). If UGT85E1 can also glycosylate cytokinins, it would result in decreased cytokinins and increased ABA responses in overexpression lines, which is consistent with our observation in this study. However, no significant catalytic activity of UGT85E1 was found toward typical cytokinins such as trans-zeatin, cis-zeatin, and isopentenyl adenine in our biochemical assays (data not shown). We also detected some other secondary natural products related to abiotic stresses, for instance, anthocyanins, flavonoids, phenolic acids, phenylpropanoids, auxins, ABA, etc. But still, no catalytic activity was found under our experimental conditions. Clearly, it requires further investigation into this aspect. The identification of substrate(s) would provide useful cues into the complex mechanism of UGT85E1 in plant abiotic stress responses.

\section{MATERIALS AND METHODS}

\section{Plant Materials and Growth Conditions}

The WT rice plant and also the genetic background of the transgenic rice plants were ZH11 (Oryza sativa L. subsp. japonica cv. Zhonghua No.11). Rice seedlings were hydroponically grown or soil-grown in a growth chamber under standard growth conditions. For Arabidopsis thaliana, the ecotype Columbia 0 (Col-0) was used as wild type and transformed by the constructed plasmids. Seeds were sown on Murashige and Skoog (MS) medium, cold-treated for 3 days at $4^{\circ} \mathrm{C}$, and transferred to controlled growth chamber under $16 \mathrm{~h}$ light/ $8 \mathrm{~h}$ dark condition with a fluency rate of $100 \mu \mathrm{mol} / \mathrm{s} / \mathrm{m}^{2}$ of white light (produced by cool-white fluorescent lamps) at $22^{\circ} \mathrm{C}$.

\section{Vector Construction and Plant Transformation}

To construct the rice overexpression vector, the full-length cDNA (1,461 bp) of UGT85E1 were amplified and cloned into pUN1301 binary vector under the control of maize ubiquitin promoter. For the ectopic expression of UGT85E1 in Arabidopsis, the 
full-length cDNA of UGT85E1 were cloned into a pBI121 binary vector under the control of CaMV 35S promoter. For the knocking-out of UGT85E1 in rice, the CRISPR/Cas9 BGK01 kit was purchased from Biogle Biotechnology Company (Hanghzhou, China) (http://www.biogle.cn) and the knockingout vector was constructed according to the instructions. Rice transformation was carried out by the Agrobacterium-mediated method (Hiei et al., 1994), and the flower dipping method was used for Arabidopsis transformation (Clough and Bent, 1998).

\section{Stress Assays of the Transgenic Plants}

For the hydroponic treatment, rice seedlings grown for 3 days were treated with $150 \mathrm{mM}$ mannitol for 1 week. Non-treated seedlings were used as the control. For the drought stress treatment of soil-grown seedlings, uniformly growing seedlings were transplanted to the soil and grown for 4 weeks under normal irrigation conditions. Then, irrigation was stopped for about 10 days and re-irrigation was provided. After the recovery for 2 days, pictures of growth phenotypes were taken and the survival ratio (the number of surviving plants over the total number of treated plants in the pot) of each line was calculated. For oxidative stress tolerance assays, leaf disks from 3-weekold seedlings were soaked into $\mathrm{H}_{2} \mathrm{O}_{2}$ or MV according to the indicated concentrations for 5 days, those leaf discs soaked in water were used as control.

To test the ABA sensitivity at the germination stage, seeds of the UGT85E1 transgenic lines were germinated on 6-well plates containing $5 \mu \mathrm{M}$ or $10 \mu \mathrm{M}$ ABA, the deionized water was used as the control. These seeds were germinated under $10 \mathrm{~h}$ light $/ 14 \mathrm{~h}$ dark condition at $28^{\circ} \mathrm{C}$ in a growth chamber for 5 days, and then the root length of each seedling was measured.

For the drought treatment of UGT85E1 transgenic Arabidopsis, 3-week-old soil-grown seedlings were kept without irrigation for 6 days and then again provided with watering. After recovery for $24 \mathrm{~h}$, the survival rate was calculated and growth performance was photographed.

\section{Measurement of Stomatal Closure in Response to Drought Treatment}

The detached leaves of 3 -week-old rice were placed in the air for $2 \mathrm{~h}$. Water is naturally lost to promote stomatal closure. Firstly, the leaves were fixed with $2.5 \%$ glutaraldehyde fixator at $4^{\circ} \mathrm{C}$ for $12-16 \mathrm{~h}$, and then washed with $1 \times$ phosphate buffered saline (PBS) for 5 times; Secondly, gradient ethanol dehydration, and then drying the samples in the dryer, spraying gold in ion sputtering device, finally a scanning electron microscope (FEI Quanta250 FEG, Oregon, USA) was used to observe and take photos.

\section{Determination of ABA Content}

Leaves of 2-week-old UGT85E1 transgenic rice and WT plants were detached and kept in the air for $2 \mathrm{~h}$. Approximately $50 \mathrm{mg}$ of leaf tissues were collected and rapidly frozen with liquid nitrogen, followed by homogenizing the sample in PBS (PH7.4). Samples were centrifuged for $20 \mathrm{~min}$, and the supernatant was collected carefully for determination of ABA. The ABA concentration was assayed according to the instructions provided by the plant hormone abscisic acid ELISA Kit (Shanghai Fusheng Industrial Co., Ltd, Catalog number: A112641-96T, Shanghai, China).

\section{Physiological Measurements}

For calculation of water loss rate, the detached leaves of the 3week-old rice plants were firstly weighed to record the fresh weight $(\mathrm{FW})$, and then the samples were dehydrated in the air. The samples were weighed every $20 \mathrm{~min}$ to record the dry weight (DW; Xiong et al., 2014). Ten plants of each line were used in each replicate with three replicates. The water loss rate is defined as the $(\mathrm{FW}-\mathrm{DW}) / \mathrm{FW} \times 100 \%$. For the determination of $\mathrm{H}_{2} \mathrm{O}_{2}$ accumulation under drought stress, 3-week-old rice plants or 4-week-old Arabidopsis plants were treated with $250 \mathrm{mM}$ mannitol for $24 \mathrm{~h}$. The leaves were subjected to $0.1 \%$ DAB staining for $16 \mathrm{~h}(\mathrm{pH}=5.8)$, followed by incubation in the de-staining buffer (ethanol/lactic acid/glycerol=3:1:1; ThordalChristensen et al., 1997). Similarly, NBT-staining for superoxide detection was conducted as described by Wohlgemuth et al. (2002).

To determine the content of proline and soluble sugar, the detached leaves of 4-week-old rice plants were dehydrated at room temperature for $1 \mathrm{~h}$, the non-treated plants were served as control $0.1 \mathrm{~g}$ of leaves were homogenized with $2 \mathrm{ml}$ $3 \%$ sulfosalicylic acid and followed by centrifugation. $1 \mathrm{ml}$ supernatant was taken and an equal volume of glacial acetic acid and ninhydrin were added, followed by inoculation in boiling water bath for $60 \mathrm{~min}$. After cooling, $3 \mathrm{ml}$ toluene was added and the content of proline was determined at $520 \mathrm{~nm}$ (Nakazawa et al., 1982). Similarly, to measure the content of soluble sugar, $0.1 \mathrm{~g}$ of the harvested leaves were homogenized in $2 \mathrm{ml}$ distilled water and boiled for $30 \mathrm{~min}$, followed by centrifugation. About $1 \mathrm{ml}$ supernatant was taken, and $2 \mathrm{ml}$ anthrone and $2 \mathrm{ml}$ sulfuric acid were added, followed by a full mix and inoculation in boiling water bath for $10 \mathrm{~min}$, then soluble sugar was measured at $620 \mathrm{~nm}$ (Morris, 1948).

\section{RNA Extraction and qRT-PCR Analysis}

For analyzing the induced expression of UGT85E1, 2-weekold hydroponically grown rice seedlings were subjected to treatments with $200 \mathrm{mM}$ mannitol or $100 \mu \mathrm{M} \mathrm{ABA}$, and the leaf tissues were harvested at $0,6,12,24$, and $48 \mathrm{~h}$ time points. For detecting expression levels of the ABA biosynthesis and signal genes, ROS scavenging enzyme genes, and stressrelated genes under stress conditions, 2-week-old hydroponically grown rice seedlings or Arabidopsis seedlings were treated with $250 \mathrm{mM}$ mannitol for $12 \mathrm{~h}$, non-treated materials were used as the control. All these harvested leaf samples were then rapidly frozen in liquid nitrogen and stored at $80^{\circ} \mathrm{C}$ for further expression analysis.

Total RNA was extracted from plant samples using the Trizol reagent (TaKaRa, Japan). Reverse transcription was performed using a Prime Script RT reagent kit (TaKaRa). qRT-PCR was performed with a CFX Connect Thermal Cycling System (Bio-Rad, USA) using a SYBR Green PCR Master Mix kit (TaKaRa). The gene expression 
levels were normalized with the reference gene OsActin1 in rice and the reference gene AtActin2 in Arabidopsis. The fold-change in the expression of each target gene was calculated by using the $2^{-\Delta \Delta C t}$ method. Primer information for the qRT-PCR assay is included in Supplementary Table 1.

\section{Statistical Analysis}

All data in this work were obtained from at least three biological replicates with three technical replicates each. Statistically significant differences were determined by one-way ANOVA, followed by Tukey's test $(P<0.05)$.

\section{ACCESSION CODES}

The gene sequence data in this study can be found in the Rice Annotation Project Database (RAP-DB, http://rapdb.dna. affrc.go.jp/) and Arabidopsis Database (TAIR, https://www. arabidopsis.org) under the following accession numbers:

UGT85E1 (Os02g0755500), OsABA1 (Os01g0737800), OsABA2 (Os03g0810800), OsABA4 (Os01g0128300), OsNCED1 (Os02g0704000), OsbZIP23 (Os02g0766700), OsABI5 (Os01g0859300), OsRAB16 (Os01g0702500), OsRAB21 (Os11g0454300), OsDREB2B (Os02g0656600), OsDREB1A (Os09g0522200), OsAPX2 (Os07g0694700), OsCATA (Os02g0115700), OsCATB (Os06g0727200), OsFeSODb (Os06g0143000), OsActin1 (Os03g0718100), AtRD29A (AT5G52310), AtRD29B (AT5G52300), AtDREB1A (AT4G25480), AtDREB1B (AT4G25490), AtActin2 (AT3G18780).

\section{REFERENCES}

Ali, J., Jewel, Z. A., Mahender, A., Anandan, A., Hernandez, J., and Li, Z. K. (2018). Molecular genetics and breeding for nutrient use efficiency in rice. Int. J. Mol. Sci. 19:E1762. doi: 10.3390/ijms19061762

Bowles, D., Isayenkova, J., Lim, E. K., and Poppenberger, B. (2005). Glycosyltransferases: managers of small molecules. Curr. Opin. Plant Biol. 8, 254-263. doi: 10.1016/j.pbi.2005.03.007

Bowles, D., Lim, E. K., Poppenberger, B., and Vaistij, F. E. (2006). Glycosyltransferases of lipophilic small molecules. Annu. Rev. Plant Biol. 57, 567-597. doi: 10.1146/annurev.arplant.57.032905.105429

Caputi, L., Malnoy, M., Goremykin, V., Nikiforova, S., and Martens, S. (2012). A genome-wide phylogenetic reconstruction of family $1 \mathrm{udp}$-glycosyltransferases revealed the expansion of the family during the adaptation of plants to life on land. Plant J. 69, 1030-1042. doi: 10.1111/j.1365-313X.2011.04853.x

Chen, T. T., Liu, F. F., Xiao, D. W., Jiang, X. Y., Li, P., Zhao, S. M., et al. (2020). The Arabidopsis UDP-glycosyltransferase75B1, conjugates abscisic acid and affects plant response to abiotic stresses. Plant Mol. Biol. 102, 389-401. doi: 10.1007/s11103-019-00953-4

Choudhury, S., Panda, P., Sahoo, L., and Panda, S. K. (2013). Reactive oxygen species signaling in plants under abiotic stress. Plant Signal Behav. 8:e23681. doi: $10.4161 /$ psb.23681

Clough, S. J., and Bent, A. F. (1998). Floral dip: a simplified method for Agrobacterium-mediated transformation of Arabidopsis thaliana. Plant J. 16, 735-743. doi: 10.1046/j.1365-313x.1998.00343.x

Cook, D., Fowler, S., Fiehn, O., and Thomashow, M. F. (2004). A prominent role for the $\mathrm{CBF}$ cold response pathway in configuring the low-temperature metabolome of Arabidopsis. Proc. Natl. Acad. Sci. U. S. A. 101, 15243-15248. doi: 10.1073/pnas.0406069101

\section{DATA AVAILABILITY STATEMENT}

The original contributions presented in the study are included in the article/Supplementary Material, further inquiries can be directed to the corresponding author/s.

\section{AUTHOR CONTRIBUTIONS}

$\mathrm{B}-\mathrm{kH}$ and QL conceived the experiments and wrote the paper with inputs from all co-authors. QL, G-rD, Y-qM, and S-mZ performed the research. QL, XL, X-kL, and Y-jL analyzed the data. All authors contributed to the article and approved the submitted version.

\section{FUNDING}

This research was supported by grants from the National Natural Science Foundation of China (No. 31970290 to B-kH).

\section{ACKNOWLEDGMENTS}

We thank Sen Wang, Haiyan Yu, and Xiaomin Zhao from the State Key Laboratory of Microbial Technology of Shandong University for their assistance in the micro-imaging of the scanning electron microscopy (SEM) analysis.

\section{SUPPLEMENTARY MATERIAL}

The Supplementary Material for this article can be found online at: https://www.frontiersin.org/articles/10.3389/fpls.2021. 790195/full\#supplementary-material

Daszkowska-Golec, A., and Szarejko, I. (2013). Open or close the gate-stomata action under the control of phytohormones in drought stress conditions. Front. Plant Sci. 4, 138-153. doi: 10.3389/fpls.2013.00138

Dong, N. Q., Sun, Y. W., Guo,T., Shi, C. L., Zhang, Y. M., Kan, Y., et al. (2020). UDP-glucosyltransferase regulates grain size and abiotic stress tolerance associated with metabolic flux redirection in rice. Nat. Commun. 11, 2629-2644. doi: 10.1038/s41467-020-16403-5

Hansen, H., and Dörffling, K. (2003). Root-derived trans-zeatin riboside and abscisic acid in drought-stressed and rewatered sunflower plants: interaction in the control of leaf diffusive resistance. Funct. Plant Biol. 30, 365-375. doi: 10.1071/FP02223

Hiei, Y., Ohta, S., Komari, T., and Kumashiro, T. (1994). Efficient transformation of rice (Oryza sativa L.) mediated by Agrobacterium and sequence analysis of the boundaries of the T-DNA. Plant J. 6, 271-282. doi: 10.1046/j.1365-313X.1994.6020271.x

Hoque, M. A., Banu, M. N. A., Nakamura, Y., Shimoishi, Y., and Murata, Y. (2008). Proline and glycinebetaine enhance antioxidant defense and methylglyoxal detoxification systems and reduce $\mathrm{NaCl}$-induced damage in cultured tobacco cells. J. Plant Physiol. 165, 813-824. doi: 10.1016/j.jplph.2007.07.013

Hou, B., Lim, E. K., Higgins, G. S., and Bowles, D. J. (2004). N-glucosylation of cytokinins by glycosyltransferases of Arabidopsis thaliana. J. Biol. Chem. 279, 47822-47832. doi: 10.1074/jbc.M409569200

$\mathrm{Hu}, \mathrm{H} . \mathrm{H}$., and Xiong, L. Z. (2014). Genetic engineering and breeding of drought-resistant crops. Annu. Rev. Plant Biol. 65, 715-741. doi: 10.1146/annurev-arplant-050213-040000

Huang, X. X., Zhu, G. Q., Liu, Q., Chen, L., Li, Y. J., and Hou, B. K. (2018). Modulation of plant salicylic acid-associated immune responses via glycosylation of dihydroxybenzoic acids. Plant Physiol. 176, 3103-3119. doi: 10.1104/pp.17.01530 
Jeon, J., Kim, N. Y., Kim, S., Kang, N. Y., Novák, O., Ku, S. J., et al. (2010). A subset of cytokinin two-component signaling system plays a role in cold temperature stress response in Arabidopsis. J. Biol. Chem. 285, 23371-23386. doi: 10.1074/jbc.M109.096644

Jiang, M., and Zhang, J. (2002). Water stress-induced abscisic acid accumulation triggers the increased generation of reactive oxygen species and up-regulates the activities of antioxidant enzymes in maize leaves. J. Exp. Bot. 53, 2401-2410. doi: $10.1093 / \mathrm{jxb} / \mathrm{erf} 090$

Jin, S. H., Ma, X. M., Kojima, M., Sakakibara, H., Wang,Y. W., and Hou, B.K. (2013). Overexpression of glucosyltransferase UGT85A1 influences transzeatin homeostasis and trans-zeatin responses likely through O-glucosylation. Planta 237, 991-999. doi: 10.1007/s00425-012-1818-4

Lee, S. C., and Luan, S. (2012). ABA signal transduction at the crossroad of biotic and abiotic stress responses. Plant Cell Environ. 35, 53-60. doi: 10.1111/j.1365-3040.2011.02426.x

Lesk, C., Rowhani, P., and Ramankutty, N. (2016). Influence of extreme weather disasters on global crop production. Nature 529, 84-87. doi: 10.1038 /nature 16467

Li, P., Li, Y. J., Zhang, F. J., Zhang, G. Z., Jiang, X. Y., Yu, H. M., et al. (2017). The Arabidopsis UDP-glycosyltransferases UGT79B2 and UGT79B3, contribute to cold, salt and drought stress tolerance via modulating anthocyanin accumulation. Plant J. 89, 85-103. doi: 10.1111/tpj.13324

Li, W., Li, M., Zhang, W., Welti, R., and Wang, X. (2004). The plasma membranebound phospholipase $\mathrm{D} \delta$ enhances freezing tolerance in Arabidopsis thaliana. Nat. Biotechnol. 22, 427-433. doi: 10.1038/nbt949

Li, Y. J., Li, P., Wang, T., Zhang, F. J., Huang, X. X., and Hou, B. K. (2018). The maize secondary metabolism glycosyltransferase UFGT2 modifies flavonols and contributes to plant acclimation to abiotic stresses. Ann. Bot. 122, 1203-1217. doi: 10.1093/aob/mcy123

Lim, E. K., and Bowles, D. J. (2004). A class of plant glycosyltransferases involved in cellular homeostasis. EMBO J. 23, 2915-2922. doi: 10.1038/sj.emboj.7600295

Liu, Z., Yan, J. P., Li, D. K., Luo, Q., Yan, Q., Liu, Z. B., et al. (2015). UDP-glucosyltransferase71C5, a major glucosyltransferase, mediates abscisic acid homeostasis in Arabidopsis. Plant Physiol. 167, 1659-1670. doi: 10.1104/pp.15.00053

Milla, M. A. R., Maurer, A., Huete, A. R., and Gustafson, J. P. (2003). Glutathione peroxidase genes in Arabidopsis are ubiquitous and regulated by abiotic stresses through diverse signaling pathways. Plant J. 36, 602-615. doi: 10.1046/j.1365-313X.2003.01901.x

Morris, D. L. (1948). Quantitative determination of carbohydrates with Dreywood's anthrone reagent. Science 107, 254-255. doi: $10.1126 /$ science.107.2775.254

Nakabayashi, R., Mori, T., and Saito, K. (2014). Alternation of flavonoid accumulation under drought stress in Arabidopsis thaliana. Plant Signal Behav. 9:e29518. doi: 10.4161/psb.29518

Nakazawa, K., Tanaka, H., and Arima, M. (1982). Rapid, simultaneous and sensitive determination of free hydroxyproline and proline in human serum by high-performance liquid chromatography. J. Chromatogr. Biomed. Appl. 233, 313-316. doi: 10.1016/S0378-4347(00)81759-4

Nguyen, K. H., Ha, C. V., Nishiyama, R., Watanabe, Y., Leyva-González, A. M., and Fujita, Y. (2016). Arabidopsis type B cytokinin response regulators ARR1, ARR10, and ARR12 negatively regulate plant responses to drought. Proc. Natl. Acad. Sci. U. S. A. 11, 3090-3095. doi: 10.1073/pnas.1600399113

Nishiyama, R., Watanabe, Y., Fujita, Y., Le, D. T., Kojima, M., Werner, T., et al. (2011). Analysis of cytokinin mutants and regulation of cytokinin metabolic genes reveals important regulatory roles of cytokinins in drought, salt and abscisic acid responses, and abscisic acid biosynthesis. Plant Cell 23, 2169-2183. doi: 10.1105/tpc.111.087395

Schachtman, D. P., and Goodger, J. Q. D. (2008). Chemical root to shoot signaling under drought. Trends Plant Sci. 13, 281-287. doi: $10.1016 / j$. tplants. 2008.04 .003

Szabados, L., and Savoure, A. (2010). Proline: a multifunctional amino acid. Trends Plant Sci. 15, 89-97. doi: 10.1016/j.tplants.2009.11.009

Thordal-Christensen, H., Zhang, Z., Wei, Y., and Collinge, D. B. (1997). Subcellular localization of $\mathrm{H}_{2} \mathrm{O}_{2}$ in plants. $\mathrm{H}_{2} \mathrm{O}_{2}$ accumulation in papillae and hypersensitive response during the barley-powdery mildew interaction. Plant $J$. 11, 1187-1194. doi: 10.1046/j.1365-313X.1997.11061187.x

Tognetti, V. B., Bielach, A., and Hrtyan, M. (2017). Redox regulation at the site of primary growth: auxin, cytokinin and ROS crosstalk. Plant Cell Environ. 40, 2586-2605. doi: $10.1111 /$ pce.13021
Tognetti, V. B., Van Aken, O., Morreel, K., Vandenbroucke, K., van de Cotte, B., De Clercq, D., et al. (2010). Perturbation of indole-3-butyric acid homeostasis by the UDP-glucosyltransferase UGT74E2 modulates Arabidopsis architecture and water stress tolerance. Plant Cell 22, 2660-2679. doi: $10.1105 /$ tpc. 109.071316

Van Breusegem, F., and Dat, J. F. (2006). Reactive oxygen species in plant cell death. Plant Physiol. 141, 384-390. doi: 10.1104/pp.106.078295

Verslues, P. E., Kim, Y., and Zhu, J. (2007). Altered ABA, proline and hydrogen peroxide in an Arabidopsis glutamate: glyoxylate aminotransferase mutant. Plant Mol. Biol. 64, 205-217. doi: 10.1007/s11103-007-9145-z

Vishwakarma, K., Upadhyay, N., Kumar, N., Yadav, G., Singh, J., Mishra, R. K., et al. (2017). Abscisic acid signaling and abiotic stress tolerance in plants: a review on current knowledge and future prospects. Front. Plant Sci. 8, 161-173. doi: $10.3389 /$ fpls.2017.00161

Watkins, J. M., Chapman, J. M., and Muday, G. K. (2017). Abscisic acid-induced reactive oxygen species are modulated by flavonols to control stomata aperture. Plant Physiol. 175, 1807-1825. doi: 10.1104/pp.17.01010

Wohlgemuth, H., Mittelstrass, K., Kschieschan, S., Bender, J., and Sandermann, H. J. (2002). Activation of an oxidative burst is a general feature of sensitive plants exposed to the air pollutant ozone. Plant Cell Environ. 25, 717-726. doi: 10.1046/j.1365-3040.2002.00859.x

Wong, C. E., Li, Y., Labbe, A., Guevara, D., Nuin, P., Whitty, B., et al. (2006). Transcriptional profiling implicates novel interactions between abiotic stress and hormonal responses in Thellungiella, a close relative of Arabidopsis. Plant Physiol. 140, 1437-1450. doi: 10.1104/pp.105.070508

Xie, H., Wan, Z., Li, S., and Zhang, Y. (2014). Spatiotemporal production of reactive oxygen species by NADPH oxidase is critical for tapetal programmed cell death and pollen development in Arabidopsis. Plant Cell 26, 2007-2023. doi: $10.1105 /$ tpc. 114.125427

Xiong, H., Li, J., Liu, P., Duan, J., Zhao, Y., Guo, X., et al. (2014). Overexpression of OsMYB48-1, a novel MYB-related transcription factor, enhances drought and salinity tolerance in rice. PLoS ONE 9:e92913. doi: 10.1371/journal.pone.0092913

Xu, N., Chu, Y., Chen, H., Li, X., Wu, Q., Jin, L., et al. (2018). Rice transcription factor OsMADS25 modulates root growth and confers salinity tolerance via the ABA-mediated regulatory pathway and ROS scavenging. PLoS Genet. 14:e1007662. doi: 10.1371/journal.pgen.1007662

Ye, N. H., Zhu, G. H., Liu, Y. G., Li, Y. X., and Zhang, J. H. (2011). ABA controls $\mathrm{H}_{2} \mathrm{O}_{2}$ accumulation through the induction of OsCATB in rice leaves under water stress. Plant Cell Physiol. 52,689-698. doi: 10.1093/pcp/pcr028

You, J., and Chan, Z. L. (2015). ROS regulation during abiotic stress responses in crop plants. Front. Plant Sci. 6, 1092-1110. doi: 10.3389/fpls.2015.01092

Zhang, Z. G., Zhang, Q., Wu, J. X., Zheng, X., Zheng, S., Sun, X. H., et al. (2013). Gene knockout study reveals that cytosolic ascorbate peroxidase 2(OsAPX2) plays a critical role in growth and reproduction in rice under drought, salt and cold stresses. PLOS ONE 8:e57472. doi: 10.1371/journal.pone.005 7472

Živanović, B., Komić, S. M., Tosti, T., Vidović. M., Prokić, L., and VeljovićJovanović, S. (2020). Leaf soluble sugars and free amino acids as important components of abscisic acid-mediated drought response in tomato. Plants 9:1147. doi: 10.3390/plants9091147

Conflict of Interest: The authors declare that the research was conducted in the absence of any commercial or financial relationships that could be construed as a potential conflict of interest.

Publisher's Note: All claims expressed in this article are solely those of the authors and do not necessarily represent those of their affiliated organizations, or those of the publisher, the editors and the reviewers. Any product that may be evaluated in this article, or claim that may be made by its manufacturer, is not guaranteed or endorsed by the publisher.

Copyright () $2021 \mathrm{Liu}$, Dong, Ma, Zhao, Liu, Li, Li and Hou. This is an open-access article distributed under the terms of the Creative Commons Attribution License (CC BY). The use, distribution or reproduction in other forums is permitted, provided the original author(s) and the copyright owner(s) are credited and that the original publication in this journal is cited, in accordance with accepted academic practice. No use, distribution or reproduction is permitted which does not comply with these terms. 